\title{
Vitamin-D-Gabe beschleunigt die Sputumkonversion bei Tbc nicht
}

Fragestellung: Kann die Zeit bis zur Konversion der Sputumkultur bei Patienten mit aktiver Tuberkulose durch Vitamin-DSupplementierung zur Standardbehandlung reduziert werden?

Hintergrund: Einige randomisierte Studien, die über die Wirksamkeit oder Sicherheit einer Vitamin-D-Supplementierung als Zusatz zur Standardbehandlung einer Tuberkulose berichtet haben, sind bereits publiziert, allerdings mit hoher Variabilität in Methode, Dosierung und Ergebnissen. Angesichts der In-vitroHinweise auf einen Nutzen und wenig Anhalt für toxische Wirkungen und der niedrigen Kosten schien nun eine klinische Studie zur Verwendung von Vitamin D gerechtfertigt. Vitamin D hat immunmodulatorische Wirkungen, die bei der Beseitigung einer mykobakteriellen Infektion

Daley P, Jagannathan V, John KR et al. Adjunctive vitamin D for treatment of active tuberculosis in India: a randomised, double-blind, placebo-controlled trial. Lancet Infect Dis 2015;15:528-34. helfen könnten.

Patienten und Methoden: Diese randomisierte doppelblinde placebokontrollierte Überlegenheitsstudie wurde an 13 Standorten in Indien durchgeführt. Nicht vorbe- handelte Patienten mit Lungentuberkulose, die sputumausstrichpositiv und HIV-negativ waren, wurden zufällig (1:1) einer Standard-Tuberkulosebehandlung mit entweder hochdosierten oraler Vitamin-D3-Supplementierung (vier Dosen zu 2,5 mg in Woche 0, 2, 4 und 6) bzw. mit Placebo zugeteilt. Weder die Patienten noch die klinischen und Laboruntersucher kannten die Behandlungszuordnung. Primärer Zielparameter für die Wirksamkeit war die Zeit bis zur Konversion der Sputumkultur. Analysiert wurde mit modifiziertem Intention-to-treat-Verfahren.

Ergebnisse: 247 Studienteilnehmer wurden den Behandlungsgruppen zugeteilt und 211 in die primäre Wirksamkeitsanalyse einbezogen. Die Zeit bis zur Kulturkonversion betrug 43,0 Tage (Median; 95\%-KI 33,3-52,8) in der Vitamin-D-versus 42,0 Tage $(33,9-50,1)$ in der Placebogruppe (log-rank $p=0,95)$. Drei Patienten (2\%) starben in der Vitamin-D- und einer (1\%) in der Placebogruppe; keiner der Todesfälle wurde der Studienmedikation zugeschrieben. Keine Patienten hatten Hyperkalzämie.

Schlussfolgerung: Die Befunde zeigen, dass Vitamin-D-Supplementierung die Zeit bis zur Konversion der Sputumkultur nicht reduziert.

\section{- Kommentar von Prof. Dr. med. Klaus Mörike}

\section{Rolle von Vitamin D in der Tuberkulose-Therapie noch unklar}

Die Frage nach der Wirkung von Vitamin D bei Tbc kann noch nicht abschließend beantwortet werden. Wie eine zuvor in London durchgeführte Studie [1] ergab auch die vorliegende Untersuchung - nun in einem Land mit endemischer Tuberkulose, in dem Vitamin-D-Konzentrationen wegen Sonnenlichtexposition und Ernährung anders sein können - negative

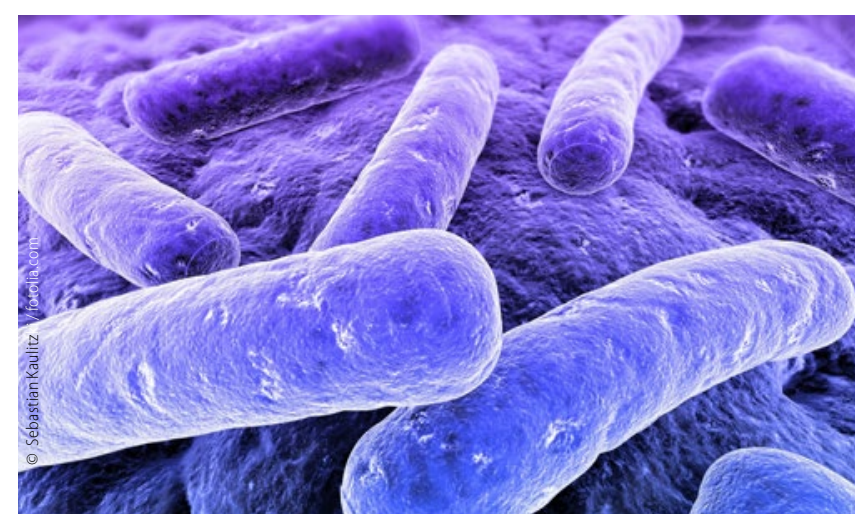

Mycobacterium tuberculosis wurde 1882 von Robert Koch entdeckt und erstmals isoliert.
Ergebnisse. Die Autoren der Studie bzw. des Begleitkommentars [2] schlagen vor, diese beiden Studien in einer Metaanalyse zu kombinieren und die Rolle von Vitamin D bei Prävention oder Reaktivierung einer Tuberkulose in weiteren Studien zu untersuchen. Die Forschung geht weiter.

\section{Literatur}

1. Martineau AR, Timms PM, Bothamley GH et al. High-dose vitamin D(3) during intensive-phase antimicrobial treatment of pulmonary tuberculosis: a double-blind randomised controlled trial. Lancet 2011; 377(9761): 242-50.

2. Cegielski $P$, Vernon A: Tuberculosis and vitamin D: what's the rest of the story? Lancet Infect Dis 2015; 15: 489-90.

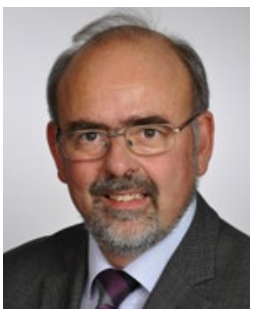

Prof. Dr. med. Klaus Mörike

Universitätsklinikum Tübingen

Abteilung Klinische Pharmakologie

Auf der Morgenstelle 8

72076 Tübingen

klaus.moerike@med.uni-tuebingen.de 